\title{
ASSISTÊNCIA DO ENFERMEIRO A MULHERES COM INDÍCIOS DE DEPRESSÃO PÓS-PARTO
}

\author{
ASSISTANCE OF NURSE TO WOMEN WITH SIGNS OF POSTPARTUM \\ DEPRESSION
}

\author{
Mirna Fernanda Miguel, Charlene Oliveira Ramos, Samylla Maira Costa Siqueira, Tânia \\ Christine Ferreira Bispo
}

Centro Universitário Jorge Amado

\begin{abstract}
The postpartum depression is characterized by the sum of depressive behaviors after the birth of a child. It start generally between $4^{\text {th }}$ and $8^{\text {th }}$ week after birth, reaching the apex in the first six months. The objective is to describe the assistance of nurse to women with signs of postpartum depression. It's an integrative literature review, carried out in March 2017 in the databases Latin American and Caribbean Health Sciences (LILACS) and Scientific Electronic Library (SciELO). Were used the descriptors "Postpartum Depression, Nursing Care and Nursing" and selected four articles. Were cited actions as host, early identification of risks, development of strategies to confrontations the problem and adaptation to puerperium and advice. Furthermore, were mentioned the necessity of familiar support to face this problem. The nurse has an important role in assistance to women with signs of postpartum depression. So, this professional must be able to recognize early signs and risk factors, in order to act and avoid the progression of the cases.
\end{abstract}

Key words: Nursing Care, Women's Health, Nursing.

\section{Resumo}

Qualifica-se a depressão pós-parto pela soma de comportamentos depressivos após o nascimento de um filho. Estes se iniciam, geralmente, entre a 4a e a 8a semana após o parto, atingindo seu ápice nos seis primeiros meses. 0 objetivo é descrever a assistência da enfermeira a mulheres com indícios de DPP. Trata-se de uma revisão integrativa da literatura, realizada em março de 2017 nas bases de dados Literatura Latino Americana e do Caribe em Ciências da Saúde (LILACS) e Scientific Electronic Library (SciELO). Foram utilizados os descritores "Depressão pósparto, assistência de enfermagem $e$ enfermagem" e selecionados quatro artigos. Destacaram-se ações como acolhimento, identificação precoce de riscos, desenvolvimento de estratégias para enfrentamento e adaptação ao puerpério e aconselhamento. Ademais, referiu-se a necessidade de suporte familiar no enfrentamento deste problema. A enfermeira tem um papel fundamental na assistência prestada à mulher com indícios de DPP. Assim, ela deve estar apta a reconhecer sinais e fatores de riscos precocemente, de modo a intervir $e$ evitar a progressão dos casos.

Palavras chave: Assistência de Enfermagem, Saúde da Mulher, Enfermagem. 


\section{Introdução}

O ciclo gravídico é caracterizado como uma fase marcada por modificações físicas, psicológicas e sociais para a mulher. Considerando que a gestação acarreta em um único momento transformações biológicas e psicossociais, esse período é considerado propício para o desencadeamento de distúrbios psiquiátricos, a exemplo da depressão pós-parto $(\mathrm{DPP})^{1-2}$.

A DPP é considerada um problema de saúde pública, isso porque atinge tanto a saúde da mãe, quanto o processo de nascimento, crescimento e desenvolvimento da criança, uma vez que tal manifestação é responsável por sofrimento intenso, incapacidades e redução da responsabilidade materna no que diz respeito à capacidade de satisfazer às necessidades da criança $^{3-4}$.

Este problema afeta mulheres em todo o mundo, com ênfase nos países em desenvolvimento. A Organização Mundial de Saúde $(\mathrm{OMS})^{4}$ revela que uma recente metanálise demonstrou que nestes países cerca de $20 \%$ das mães vivenciam a DPP, sendo o suicídio uma importante causa de morte entre gestantes e puérperas. No que concerne especificamente ao Brasil, estima-se que a DPP atinja de 10 a $15 \%$ das parturientes, fortalecendo a sua caracterização de problema de saúde pública e, por conseguinte, requerendo estratégias de prevenção e terapêutica ${ }^{3}$.

Qualifica-se a DPP pela soma de comportamentos depressivos após o nascimento de um filho. Estes se iniciam, geralmente, entre a 4a e a 8a semana após o parto, atingindo seu ápice nos seis primeiros meses. Dentre os sinais e sintomas apresentados pela mulher, destacam-se mudança de humor; choro frequente; sentimento de culpa; alterações no sono, apetite, libido e no nível de funcionamento mental; e medo de machucar o filho ${ }^{5}$.

Vários são os fatores considerados como risco para a DPP, dentre os quais pode-se citar: ausência de companheiro ou relacionamento com pouco tempo, gravidez não planejada, complicações na gestação e puerpério, história pregressa de distúrbios mentais por parte da gestante, baixa escolaridade e múltiplas gestações e partos ${ }^{6-7}$. Em um estudo transversal realizado com puérperas no Espírito Santo, os autores $^{7}$ aventaram que as mulheres que apresentaram mais depressão foram aquelas com menor escolaridade, maior número de gestações e partos, maior número de filhos vivos e relacionamentos recentes, asseverando a associação entre a DPP com fatores sociais.

Estes mesmos autores ${ }^{7}$ destacam a importância da detecção precoce desta problemática, de forma a interferir precocemente, evitando as consequências desta patologia às puérperas. Dentre os profissionais que podem contribuir na assistência à mulher com indícios de DPP destaca-se a enfermeira, uma vez que esta acompanha a gestante desde o pré-natal e após o nascimento do concepto, podendo identificar precocemente traços emocionais, fatores sociais e comportamentais que sugiram a depressão ${ }^{8}$ e intervir com brevidade, de forma a oferecer suporte e garantir os devidos encaminhamentos, buscando interromper a cadeia da depressão e suas consequências.

Neste direcionamento, estudos acerca desta temática são essenciais, possibilitando às enfermeiras a compreensão sobre os aspectos que permeiam a DPP, bem como sobre a assistência a ser prestada no enfrentamento destes casos, favorecendo a prática de enfermagem baseada em evidências.

Frente ao exposto, este estudo é norteado pela seguinte questão de pesquisa: qual a assistência do enfermeiro a mulheres com indícios de DPP?. Diante disto, estabeleceu-se como objetivo descrever a assistência da enfermeira a mulheres com indícios de depressão pós-parto.

\section{Metodologia}

Trata-se de uma revisão integrativa da literatura, realizada a partir das cinco fases propostas para este tipo de estudo: elaboração do problema e pergunta de pesquisa, coleta dos dados, avaliação dos dados coletados, análise e interpretação das informações e apresentação dos resultados ${ }^{9}$.

A coleta dos dados ocorreu em março de 2017 nas bases de dados Literatura Latino Americana e do Caribe em Ciências da Saúde (LILACS) e Scientific Electronic Library Online (SciELO). A pesquisa nas bases foi realizada a partir dos seguintes descritores, consultados na biblioteca dos Descritores em Ciências da Saúde (DeCS): "Depressão pós-parto, assistência de enfermagem e enfermagem". Em ambas as bases, a busca ocorreu a partir do uso do operador booleano "AND", com a combinação dos descritores da seguinte forma: 1) depressão pós-parto and enfermagem; 2) assistência de 
enfermagem and depressão pós-parto.

As combinações supracitadas permitiram identificar 48 materiais: 21 na LILACS e 12 na SCIELO a partir da primeira associação e 12 na LILACS e 3 na SciELO a partir da segunda. Estes foram filtrados a partir dos seguintes critérios de inclusão: artigos com disponibilidade de texto na íntegra, publicados em português, no recorte temporal de sete anos (2010 a 2016). Foram excluídos os artigos repetidos e aqueles que não eram compatíveis com o objetivo proposto.

\section{Resultados}

A partir dos critérios supracitados, foram selecionados 23 artigos (14 na LILACS e 9 na SciELO). Estes tiveram o título e resumo lidos para identificação de compatibilidade com a temática aqui pesquisada. Ao final, foram selecionados apenas 4 artigos, cuja síntese encontra-se no Quadro 1.

Quadro 1 - Síntese dos artigos selecionados $(n=4)$ quanto ao ano, autores, base de dados, periódico e título. Salvador - BA, Brasil, 2017.

\begin{tabular}{|c|c|c|c|l|l|}
\hline ID & Ano & Autores & $\begin{array}{c}\text { Base de } \\
\text { Dados }\end{array}$ & Periódico & \multicolumn{1}{|c|}{ Título } \\
\hline $\mathbf{1}$ & 2010 & $\begin{array}{c}\text { Valença, } \\
\text { Germano }\end{array}$ & Lilacs & Enfermagem & $\begin{array}{l}\text { Prevenindo a depressão puerperal na estratégia } \\
\text { saúde da família: Ações de enfermeiro no pré- } \\
\text { natal }\end{array}$ \\
\hline $\mathbf{2}$ & 2010 & $\begin{array}{c}\text { Gomes et } \\
\text { al }^{12}\end{array}$ & Lilacs & Enfermagem & $\begin{array}{l}\text { Identificação dos fatores de risco para depressão } \\
\text { pós-parto: importância do diagnóstico precoce }\end{array}$ \\
\hline $\mathbf{3}$ & $\mathbf{2 0 1 3}$ & $\begin{array}{c}\text { Daandels, } \\
\text { arboit, Van } \\
\text { der sand }\end{array}$ & Lilacs & Enfermagem & $\begin{array}{l}\text { Produção de enfermagem sobre depressão pós- } \\
\text { parto }\end{array}$ \\
\hline $\mathbf{4}$ & $\mathbf{2 0 1 4}$ & $\begin{array}{c}\text { Freitas et } \\
\text { al }^{13}\end{array}$ & Lilacs & Enfermagem & $\begin{array}{l}\text { Alojamento conjunto em um hospital universitário: } \\
\text { depressão pós-parto }\end{array}$ \\
\hline
\end{tabular}

Foram encontrados artigos publicados em $2010(n=2), 2013(n=1)$ e $2014(n=1)$, todos na LILACS. A maioria $(n=3)$ se tratava de trabalhos originais e um foi uma revisão bibliográfica do tipo narrativa. Quanto à natureza, houve trabalhos com abordagem qualitativa $(n=3)$ e quantitativa $(n=1)$. Em se tratando do local de realização, todos foram desenvolvidos no Brasil, tendo sido observados trabalhos realizados no Ceará $(n=2)$, Rio de Janeiro $(n=1)$ e Rio Grande do Sul $(n=1)$. No que diz respeito à autoria, os autores dos três artigos são da área da Enfermagem.

Quanto à assistência da enfermeira a mulheres com indícios de DPP, a partir dos trabalhos selecionados foi possível identificar ações desenvolvidas por este profissional, assim como o apoio da família no enfrentamento desta problemática. Assim, foram criadas duas categorias para discussão: 1) Assistência da enfermeira a mulheres com indícios de depressão pós-parto; e 2) Suporte familiar a mulheres com indícios de depressão pós-parto.

\section{Assistência da enfermeira a mulheres com indícios de depressão pós-parto}

Os cuidados da enfermeira às mulheres com indícios de DPP segundo os autores selecionados se relacionaram ao acolhimento ${ }^{10}$; identificação precoce de riscos ${ }^{11-12}$; desenvolvimento de estratégias para enfrentamento, adaptação ao puerpério ${ }^{13}$; e aconselhamento ${ }^{11-12}$.

O acolhimento é caracterizado como um arranjo profissional em que há uma postura ética que implica na escuta das queixas do usuário, no reconhecimento do seu protagonismo no processo de saúde e adoecimento e no compromisso pela solução, buscando garantir o acesso e resolver os problemas, quando possível, ou ao menos fornecer direcionamentos. Assim, acolher se traduz em um compromisso de resposta às necessidades das pessoas que procuram os serviços de saúde ${ }^{14-15}$.

Os reflexos do acolhimento nos serviços de saúde se revelam no fortalecimento do elo entre usuários e profissionais, permitindo a identificação de problemas e, consequentemente, a intervenção precoce para resolvê-los. Neste contexto, destaca-se a conduta de acolhimento como uma atitude frequente para identificação da DPP ${ }^{16-17}$.

Uma pesquisa ${ }^{18}$ desenvolvida no Hospital das Clínicas de Porto Alegre com o objetivo de conhecer o significado do acolhimento para usuários do Sistema Único de Saúde (SUS) revelou que os participantes percebiam o acolhimento como um espaço de atenção, caracterizado com uma necessidade de "zelo, respeito, cortesia e diálogo", destacando a importância de a equipe de saúde estar atenta 
para responder a tais expectativas.

O acolhimento relacionado à DPP é apontado como uma competência que deve ocorrer desde o pré-natal, de forma humanizada e integral, no sentido de acolher a gestante e adotar medidas de prevenção desta alteração química sempre que possível ${ }^{10}$, além de observar os sinais precocemente. Assim sendo, destaca-se a importância de a enfermeira elaborar atividades de promoção da saúde e prevenção de agravos no transcorrer do pré-natal, estando estas focadas não somente no período gravídico, mas na saúde da mulher de forma integral ${ }^{10}$.

Em um dos estudos selecionados neste levantamento $^{10}$, o qual foi realizado com mulheres no puerpério atendidas em uma Unidade de Saúde da Família (USF) de Natal-RN, foi relatado pelas pacientes que o acolhimento e as rodas de conversa desenvolvidas pelo enfermeiro foram essenciais para alívio da DPP, revelando a importância desta prática na melhoria do quadro clínico de puérperas com tal problema.

Na prática diária dos serviços de saúde, o acolhimento e a humanização devem-se tornar uma meta, sendo alcançados por meio de atitudes e condutas relacionadas ao vínculo junto aos profissionais, sendo recomendadas ações como apresentação da enfermeira à paciente, comunicação acerca das ações e métodos de cuidado e motivação à presença do companheiro ${ }^{19}$.

No que diz respeito aos riscos para desenvolvimento da DPP, são destacados na literatura a gestação não programada/planejada, maternidade precoce, baixo nível socioeconômico, multiparidade, relação conjugal instável, colaboração insuficiente por parte dos familiares nos cuidados à criança, desocupação, impossibilidade de amamentar ao seio e distúrbios psiquiátricos anteriores ou durante a gestação ${ }^{20}$.

Corroborando tal afirmativa, uma pesquisa ${ }^{12}$ desenvolvida com puérperas de uma maternidade de referência de Fortaleza com o objetivo de identificar os fatores que podem contribuir para a DPP revelou como os principais fatores de risco encontrados para a manifestação desta problemática a maternidade na adolescência, baixa renda, baixa escolaridade e instabilidade na relação conjugal.

Autores referem que a DPP é responsável por gerar relevantes efeitos no crescimento emocional, social e mental da criança. Assim, a identificação da DPP é primordial, pois permite o tratamento do problema, de modo a preservar a criança de sequelas relacionadas a esse problema ${ }^{14}$.

Tal afirmativa é coadunada pelos achados de uma pesquisa ${ }^{21}$ conduzida em uma Unidade Básica de Saúde (UBS) paranaense, a qual revelou que uma das participantes durante a DPP desenvolveu pela filha sentimentos de desprezo e negação, referindo não ter conseguido amar a criança durante o período do distúrbio. Diante disso, os autores ${ }^{21}$ concluem que a paciente com DPP sofre com crises de raiva, stress e irritabilidade, havendo momentos de reações agressivas e recusa do bebê.

Neste direcionamento, é fundamental a investigação a respeito das experiências da mulher no decorrer da gravidez e, principalmente, como está se passando o período pós-parto. O diagnóstico da condição deprimida da mãe é essencial, embora ocasionalmente complexo, contudo indispensável ao enfrentamento do problema ${ }^{22}$. Diante disso, as equipes de saúde devem estar preparadas para identificar e cuidar de uma gestante com predisposição a evoluir para DPP, prevenindo possíveis consequências, que podem ser fatais e gerar sofrimento para a mãe, o bebê e toda a família. Assim, é necessário a preparação da enfermeira que assiste a gestante para identificação precoce dos riscos e encaminhamento da paciente para terapia, na perspectiva de bloquear esse distúrbio psíquico.

Os resultados auferidos por um estudo ${ }^{23}$ realizado em UBS de Goiânia e Fortaleza com mulheres com sinais de DPP revelou prevalência de $24,2 \%$ até $74,7 \%$ do quadro depressivo, respectivamente. Assim, os autores asseveram que quanto antes os sintomas forem apresentados, mais depressa as intervenções poderão ser executadas, classificando como de extrema importância a presença nas consultas de pré-natal e observação sistemática pela enfermeira ${ }^{23}$.

Diante disso, é imprescindível que sejam compreendidas as alterações biopsicossociais vivenciadas pelas puérperas, sendo utilizadas estratégias como atenção, comunicação e acolhimento, no sentido de permitir a criação de vínculo e, como consequência, o reconhecimento antecipado do problema, com intervenção precoce a partir de orientações durante todo o processo gravídico-puerperal. Infere-se, inclusive, que os cuidados não devem ser centrados apenas nos aspectos obstétricos, mas à saúde da mulher de forma integral ${ }^{13}$. 
Uma pesquisa ${ }^{24}$ desenvolvida com 15 enfermeiros lotados em unidades primárias à saúde de Minas Gerais revelou que os participantes compreendem a necessidade de identificar precocemente os sinais de DPP, verbalizando como estratégia o acolhimento a partir da escuta qualificada, especialmente na primeira consulta de pré-natal.

A partir do acolhimento, a enfermeira terá espaço com a paciente para implementar estratégias de enfrentamento à DPP, como o aconselhamento, definido como uma intervenção de cuidado diferenciada que, embora deva ser oferecida por terapeuta, destaca-se que pelo fato de a enfermeira conviver intensamente com a mulher durante o período gestacional e após este, esta profissional também é indicada para o desenvolvimento de tal estratégia, reconhecendo precocemente os sinais de DPP e fornecendo à paciente suporte emocional e encaminhamento à terapia ${ }^{11,15}$, uma vez que a literatura a respeito do tema aponta o aconselhamento como uma forma de evitar tal problema ${ }^{12}$.

Em uma pesquisa que avaliou o aconselhamento em comparação com cuidados usuais com pacientes com depressão atendidos em serviços de atenção primária, foi observado que após três meses de aconselhamento houve melhora no quadro depressivo das mulheres acompanhadas ${ }^{25}$, reafirmando a importância desta estratégia no cuidado à paciente com indícios de DPP.

A efetividade do suporte fornecido pela enfermeira à mulher com DPP foi revelada em uma pesquisa ${ }^{10}$ realizada em uma UBS do Rio Grande do Norte com pacientes que reconheceram o apoio da enfermeira, realizado a partir de visitas e encaminhamento ao psiquiatra. Assim, reforça-se a importância desta profissional nos cuidados à mulher com DPP.

\section{Suporte familiar a mulheres com indícios de depressão pós-parto}

Embora a enfermeira tenha função relevante no enfrentamento da DPP, esta profissional não pode atuar sozinha no controle de um problema tão complexo, sendo necessário o apoio de outros atores sociais. Neste contexto, emergiu como resultado desta categoria o apoio dos familiares da paciente com indícios de DPP ${ }^{13}$, evidenciando que a assistência à mulher com esta problemática deve incluir os cuidados familiares.

O suporte familiar é de fundamental importância por esta ser o principal contato social da paciente. Assim, a família precisa reconhecer a importância de sua função na preservação da saúde da mulher, devendo refugiar, entender, ouvir e direcionar, além de monitorar o uso dos medicamentos, quando for o caso, e acompanhar a paciente nas consultas terapêuticas e de enfermagem, considerando-se que esse distúrbio não afeta somente a mãe e o bebê, mas todos do círculo familiar.

O apoio da família é retratado em um estudo $^{26}$ realizado com familiares de paciente com DPP em um hospital de saúde mental do Distrito Federal, o qual revelou que os participantes tentam ajudar por meio de diálogo, lazer, aumento da autoestima e suporte nos cuidados com a criança. Convém destacar, contudo, que apenas $10 \%$ dos familiares entendem que se trata de um distúrbio que necessita de tratamento, acolhimento e atenção, sendo necessário a sensibilização da família por parte dos profissionais de saúde.

Diante disso, destaca-se a importância de a enfermeira envolver a família no processo, alertando-os acerca do reconhecimento de sinais e sintomas da DPP e da necessidade de ofertar suporte à paciente e comunicar a equipe possíveis sinais de piora do quadro, sendo a comunicação um processo necessário no enfrentamento deste problema ${ }^{13,27}$.

O déficit na comunicação entre a enfermagem e a família foi apontado em um estudo $^{28}$ como motivo de insatisfação por parte dos familiares, que referiram ter de procurar por diferentes grupos profissionais dentro de uma instituição hospitalar para adquirir alguma informação. Em oposição, outra pesquisa ${ }^{29}$ desenvolvida em um centro obstétrico paulista revelou que uma paciente referiu segurança e acolhimento pelo fato de as enfermeiras terem estado próximas, permitindo aos autores caracterizarem a enfermeira como peça principal na atenção à mulher e à família, sendo a responsável pela comunicação na reestruturação familiar do paciente.

A parceria entre enfermeira e família foi apontada como positiva por autores ${ }^{30}$ que conduziram um estudo com famílias no programa da saúde da família de Goiânia, referindo que a enfermeira bem qualificada consegue desempenhar ações preventivas já na gravidez e pode tornar-se um apoio diante da DPP, uma vez que as famílias referiram que tal parceria ajudou muito.

Assim, destaca-se que ao suporte da família é fundamental na atenção à mulher no período gravídico-puerperal, devendo acompanhar a 
paciente e auxiliar nos cuidados desta e de seu bebê, principalmente no primeiro ano de vida ${ }^{31}$.

\section{Conclusões}

Concluso este levantamento, reforça-se que a DPP não diagnosticada precocemente pode trazer várias consequências, podendo ou não ser fatais e afetando a todos que compõem o círculo familiar da paciente, em especial o bebê que muitas vezes é negligenciado, o que irá refletir em seu desenvolvimento.

A enfermeira é a profissional que está diretamente ligada ao cuidado à mulher. Assim, ela precisa ter embasamento técnico-científico para reconhecer precocemente os sinais e fatores de riscos que podem desencadear a depressão, além de ter um olhar criterioso não apenas aos aspectos concernentes ao período gestacional, mas à saúde integral da mulher.

Vale salientar que os cuidados não devem ser voltados apenas para a saúde do binômio como também para toda a família, envolvendo-a neste processo, uma vez que o apoio familiar é de extrema importância, colaborando como elo com a equipe, apontando sinais que possam suscitar o transtorno a fim de resolvê-lo com a maior brevidade possível.

\section{Referências}

1. Araújo NM, Salim NR, Gualda DMR, Silva LCFP. Corpo e sexualidade na gravidez. RevEscEnfermUSP2012; 46(3):552-558.

2. Cantilino A, Zambaldi CF, Sougey EB, Rennó Júnior J. Transtornos psiquiátricos no pósparto. Rev. Psiquiatr. Clín. 2010; 37(6):278-284.

3. Tolentino EC, Maximino DMF, Souto VGC. Depressão pós-parto: conhecimento sobre os sinais e sintomas em puérperas. Rev. Ciênc. Saúde Nova Esperança 2016; 14(1):59-66.

4. World Health Organization. Maternal mental health. Internet. Available in: http://www.who.int/mental_health/maternalchild/maternal_mental_health/en/. Acess in: 20 april 2017.

5. Corrêa PF, Seralha AC. A depressão pósparto e a figura materna: uma análise retrospectiva e contextual. Acta. colomb. psicol. 2015; 18(1):113-123.

6. Soares YKCS, Gonçalves NPC, Carvalho CMS. Avaliação da depressão pós-parto: prevalência e fatores associados. R. Interd. 2015; 8(4):40-46.

7. Ruschi GEC, Sun SY, Mattar R, Chambô
Filho A, Zandonade E, Lima VJ. Aspectos epidemiológicos da depressão pós-parto em amostra brasileira. RevPsiquiatr RS. 2007;29(3):274-280.

8. Brocchi SB, Bussab RSV, David V. Depressão pós-parto e habilidades pragmáticas: comparação entre gêneros de uma população brasileira de baixa renda.AudiolCommun Res, 2015; 20(3): 262-268.

9. Whittemore R, Knafl K. The integrative review: update methodology. J AdvNurs. 2005; 52(5):546-53.

10. Valença CN, Germano RM. Prevenindo a depressão puerperal na Estratégia Saúde Da Família: ações do enfermeiro no pré-natal. Rev. Rene. 2010; 11(2):129-139.

11. Daandels N, Arboit EL, Sand ICPVD. Produção de enfermagem sobre depressão pósparto. CogitareEnferm. 2013; 18(4):782-788.

12. Gomes LA, Torquato VS, Feitoza AR, Souza AR, Silva MAM, Pontes RJS.Identificação dos fatores de risco para depressão pós-parto: importância do diagnóstico precoce. Rev. Rene 2010; 11(esp):117-123.

13. Freitas DR, Vieira BDG, Alves VH, Rodrigues DP, Leão DCMRL, Cruz AFN, Azevedo CS, Gaiva MAM. Alojamento conjunto em hospital universitário: depressão pós-parto na perspectiva do enfermeiro. J. res.:fundam. care online[Internet]. Abr./Jun. 2014 [acesso em: 01 mai 2017]; 6(2):1202-1211. Disponível: http://saudepublica.bvs.br/pesquisa/resource/pt /bde-25656

14. Schmidt EB, Piccoloto NM, Muller MC.Depressão pós-parto: fatores de risco e repercussões no desenvolvimento infantil. PsicoUSF 2005; 10(1):61-68.

15. Ministério da Saúde. Cartilha do PNH: Acolhimento nas Práticas de Produção de Saúde. Brasília, DF: Ministério da Saúde; 2005.

16. Garuzi M, Achitti COM, Sato CA, Rocha AS, Spagnuolo RS. Acolhimento na Estratégia Saúde da Família: revisão integrativa. Rev Panam Salud Publica 2014; 35(2):144-149.

17. Meira BM, Pereira PAS, Silveira MFA, Gualda MR, Santos Júnior HPO. Desafios para profissionais da atenção primária no cuidado à mulher com depressão pós-parto. Texto Contexto Enferm 2015; 24(3):706-712.

18. Falk MLR, Falk JW, Oliveira FA, Motta MS. Acolhimento como dispositivo de humanização: percepção do usuário e do trabalhador em saúde. Rev. APS 2010; 13(1):4-9.

19. Ministério da Saúde. Acolhimento à demanda espontânea. Brasília, DF: Ministério da Saúde; 2005.

20. Guedes ACE, Kami CT, Cavalli LKV, 
Nicolaou SK, Hess VB, Maluf EMCP. Depressão pós-parto: incidência e fatores de risco associados. RevMed 2011; 90(3):149-154.

21. Deziderio D, Milani RG. As influências da depressão pós-parto na relação mãe-bebê. In: Anais do VIII EPCC - Encontro Internacional de Produção Científica Cesumar; 2013 out. 10-14; Maringá, Brasil. Paraná: Editora Cesumar; 2013.

22.

Schwengber

DDS, Piccinini CA. O impacto da depressão pósparto para a interação mãe-bebê. Estudos de Psicologia 2003; 8(3):403-411.

23. Boska GA, Wisniewski D, Lentsck $\mathrm{MH}$. Sintomas depressivos no período puerperal: identificação pela escala de depressão pós-parto de Edinburgh. J Nurs Health. 2016; 6(1):38-50.

24. Sobreira NAS, Pessôa CGO. Assistência de enfermagem na detecção da depressão pósparto. Revista Enfermagem Integrada 2012; 5(1):905-18.

25. Wannmacher L. Abordagem da depressão maior em idosos: medidas não medicamentosas e medicamentosas. [Internet] 2016 [acesso em: 15 mai 2017]; 1(1). Disponível em:

file://C:/Users/DSSF/Downloads/Downloads/Ab ordagem\%20idosos_F001\%20(1).pdf

26. Feitosa MP, Bohry S, Machado ER. Depressão: Família seu papel no tratamento do paciente. Encontro Revista de Psicologia 2011; 14(21):127-144.

27. Ribeiro GW, Andrade M. O papel do enfermeiro na prevenção da depressão pós-parto (DPP). Informe-se em Promoção da Saúde 2009; 5(1):7-9.

28. Casanova EG, Lopes GT. Comunicação da equipe de enfermagem com a família do paciente. RevBrasEnferm 2009; 62(6):831-836.

29. Caron OAF, Silva IA. Parturiente e equipe obstétrica: a difícil arte da comunicação. Rev Latino-am Enfermagem [Internet]. jul-ago. 2002 [acesso em: 01 mai 2017]; 10(4):485-492. Disponível em:

http://www.scielo.br/scielo.php?pid=S0104$11692002000400004 \&$ script $=$ sci_abstract $\&$ tlng $=p$ $\mathrm{t}$

30. Matão MEL, Miranda DB, Campos PHF, Oliveira LN, Martins VR. Experiência de familiares na vivência da depressão pós-parto. R. Enferm. Cent. O. Min. 2011; 1(3):283-293.

31. Melo EC, Oliveira RR, Mathias TAF. Fatores associados à qualidade do pré-natal: uma abordagem ao nascimento prematuro. RevEscEnferm USP 2015;49(4):540-549.

\section{Endereço para Correspondência}

Centro Universitário Jorge Amado

Av. Luís Viana, 6775 - Paralela, Salvador - BA

CEP.: 41745-130

e-mail: smcsiqueira@hotmail.com

Recebido em 28/06/2017

Aprovado em 09/11/2017

Publicado em 29/03/2018 